\title{
OPA1 mutations and mitochondrial DNA haplotypes in autosomal dominant optic atrophy
}

Jian Han, $P h D^{1}$, Angela J. Thompson-Lowrey, BS $S^{1}$, Alyson Reiss, $B S^{3}$, Vladimir Mayorov, $P h D^{1}$, Haomiao Jia, $P h D^{2}$, Valerie Biousse, $M D^{3,4}$, Nancy J. Newman, $M D^{3,5}$, and Michael D. Brown, PhD ${ }^{1}$

\begin{abstract}
Purpose: Autosomal dominant optic atrophy is a form of blindness, due in part to mutations affecting the mitochondrial-targeted OPA1 gene product. Both OPA1-positive and OPA1-negative families exhibit variable expressivity and incomplete penetrance. The purpose of this study was therefore to determine if the background mtDNA genotype acts as a genetic modifier for the expression of this disease. Methods: To find novel pathogenic OPA1 mutations, we performed complete OPA1 gene exon sequencing in 30 patients. To assess the possibility that mitochondrial DNA haplotype acts as a genetic modifier, we determined the mitochondrial DNA haplotype in 29 Caucasian OPA1-positive and OPA1-negative patients. Deviations in haplotype distribution between patient and control groups were determined by statistical means. Results: Seven new pathogenic OPA1 mutations were found. Most were detected in the mitochondrial targeting N-terminus or in the coiled-coil domain at the C-terminus. Mitochondrial DNA haplotype analysis indicated that the European haplogroup distribution was different between Caucasian patients and controls. Further, haplogroup J was three-fold over-represented in OPA1-negative patients.

Conclusions: Overall, our results support haploinsufficiency as a genetic mechanism in OPA1-positive cases and also suggest that mtDNA genetic background may influence disease expression in a subset of cases. Genet Med
\end{abstract} 2006:8(4):217-225.

Key Words: autosomal dominant optic atrophy, OPA1, mitochondria, mtDNA, haplotypes, genetic modifier

Autosomal dominant optic atrophy (ADOA) is a form of blindness in which patients suffer from progressive, bilateral vision loss from degeneration of retinal ganglion cells (RGCs). ADOA is clinically characterized by loss of visual acuity, central visual field defects, color vision abnormalities, and optic nerve pallor. ${ }^{1-3}$ Disease progression is typically slow and the age of onset is frequently in childhood, although it can vary significantly. ${ }^{3}$ Neuropathologically, ADOA shares end-stage features, including retinal ganglion cell death and optic nerve atrophy, with the primary mitochondrial optic neuropathy, Leber's Hereditary Optic Neuropathy (LHON), a disorder caused by point mutations in the mitochondrial DNA (mtDNA). Like LHON, sporadic cases are not uncommon.

Mutations in the OPA1 gene account for a number of ADOA cases. ${ }^{2,3}$ The OPA1 gene encodes a 960-amino acid dynaminlike GTPase protein consisting of an N-terminal mitochondrial leader sequence, a highly conserved GTPase (exons 8-16)

\footnotetext{
From the ${ }^{1}$ Department of Basic Medical Sciences; ${ }^{2}$ Department of Community Medicine, Mercer University School of Medicine, Macon, GA; ${ }^{3}$ Department of Ophthalmology; ${ }^{4}$ Department of Neurology; ${ }^{5}$ Department of Neurological Surgery, Emory University School of Medicine, Atlanta, GA.

Michael D. Brown, PhD. The Coca-Cola Company, One Coca-Cola Plaza, TEC 440, Atlanta, GA 30313.

Submitted for publication December 7, 2005.

Accepted for publication February 2, 2006.

DOI: 10.1097/01.gim.0000214299.61930.c0
}

domain, and a C-terminal coiled-coil (including exons 27 and 28) motif. ${ }^{4-6}$ It is ubiquitously expressed in the body, with highest expression in the retina and brain. ${ }^{7,8}$ Several layers of the neural retina, including the RGCs and the optic nerve, express the gene product. ${ }^{9}$ The OPA1 protein localizes to the mitochondrial inner membrane space where it is anchored to the mitochondrial inner membrane and serves a critical function in mitochondrial morphology including cristae organization and mitochondrial fusion/network dynamics. ${ }^{6,10,11}$ RNA interference studies in human and mouse cells demonstrate that disruption of OPA1 expression leads to mitochondrial fragmentation and cristae disorganization and a loss of mitochondrial membrane potential, ${ }^{6,10-12}$ potentially initiating mitochondrial-mediated apoptosis. ${ }^{11-13}$ Accordingly, Lodi et al. (2004) recently demonstrated that OPA1 mutations in ADOA patients can result in reduced in vivo ATP synthesis in skeletal muscle. ${ }^{14}$ Thus, although the precise pathophysiological mechanism for ADOA has not been elucidated, it is clear that $O P A 1$ is a mitochondrial protein crucial for optic nerve function and that perturbations in OPA1 structure or amount precipitate mitochondrial morphological and functional defects resulting in optic atrophy.

To date, at least 98 OPA1 mutations have been associated with ADOA. ${ }^{3}$ Although a deleterious mutation can occur throughout the gene, Thistleton et al. (2002) have identified three general regions where mutations have loosely clustered: the N-terminal leader sequence (exons 1-2) domain, the 
GTPase (exons 8-16) domain, and the C-terminus (exons 27-28). ${ }^{15}$ Because the majority of OPA1 mutations truncate or disrupt the normal gene product and thus represent null alleles, haploinsufficiency has been cited as the major mechanism of action. ${ }^{2,3,16}$ However, there are a number of pathogenic missense mutations and an example of semi-dominant inheritance in an OPA1 compound heterozygote, suggesting that different pathways of altered OPAl protein function or production can lead to ADOA. , $^{2,15,16}$

OPA1 mutations account for roughly one-third to twothirds of ADOA cases, and this locus heterogeneity may be responsible for the considerable phenotypic heterogeneity seen in ADOA. ${ }^{3,5,15,16}$ Other genetic loci associated with Mendelian inherited optic atrophy have been mapped including two additional autosomal dominant (OPA4 and OPA5), a variable form that can be recessive or dominant with cataracts (OPA3), and an X-linked (OPA2) optic atrophy locus. ${ }^{17-19}$ However, incomplete penetrance and variable expressivity is common even within ADOA families. Penetrance estimates range from roughly $40-90 \% .3,5,15,16$ Individuals harboring a pathogenic OPA1 mutation may be asymptomatic, showing subtle clinical signs apparent only upon careful clinical examination, or have early-onset optic atrophy. Although often considered a childhood-onset disease, age of onset is quite variable. In two recent studies, roughly $50 \%$ of genetically verified ADOA cases had an age of onset of $<20$ years. ${ }^{1,3}$ Finally, like LHON, sporadic cases are not uncommon. However, given the potential for a mild phenotype in carriers, the frequency of singleton cases would potentially be reduced if all carrier family members were clinically examined. Also like LHON, penetrance may be mutation specific. In LHON, individuals harboring the 11778A mtDNA mutation have a higher penetrance and a lower spontaneous visual recovery rate than the other two common LHON mtDNA mutations 3460A and 14484C. ${ }^{20}$ In ADOA for example, penetrance associated with the OPA1 exon 12 c. $1212+1 \mathrm{G}>\mathrm{T}$ splicing mutation is $100 \%$, yet is only $43 \%$ in families with the c. $2708 \mathrm{del}$ (TTAG) exon 27 mutation. ${ }^{5,15}$ Interestingly, the c.2708del(TTAG) variant has been found in a number of independent ADOA families, thus representing one of the few recurrent $O P A 1$ variants associated with optic atrophy.

Intra-familial variation in ADOA expression indicates that it is highly unlikely that genetic heterogeneity is soley responsible for the observed penetrance and expressivity differences seen within and between ADOA families. One possible explanation for clinical heterogeneity is the presence of one or more modifier gene(s) that may have a role in modulating OPA1 mutant expression. ${ }^{2,15}$ If this mechanism is viable, a logical candidate for a genetic modifier would be the mtDNA since both mtDNA and $O P A 1$ gene products are mitochondrial proteins, defects in which alter mitochondrial function and result in a clinically and neuropathologically similar optic atrophy. MtDNA background, or haplogroup (a collection of related haplotypes), has been suggested as a modifying element in a number of diseases, including LHON, where a certain mtDNA genotype (haplo- group J) is associated with the $10663 \mathrm{C}, 14484 \mathrm{C}$ LHON (and to a lesser extent), the $11778 \mathrm{~A}$ primary mutations. ${ }^{21-23}$

In this report, we describe complete genomic sequencing of 30 ADOA patients, yielding seven novel OPA1 pathogenic variants and a number of new single nucleotide polymorphisms (SNPS). For 29 of the patients, we also determined the mtDNA haplotype. This report therefore not only adds to the spectrum of disease-causing OPA1 mutations, but also represents the first attempt to identify a genetic modifier of ADOA.

\section{MATERIALS AND METHODS}

\section{Patients}

Thirty unrelated ADOA patients were recruited in the study. All patients were examined by one of two experienced neuroophthalmolgists (NJN, VB). The diagnosis of ADOA was based on a typical history of insidious, painless bilateral visual loss related to bilateral optic neuropathies, examination findings of bilateral diminished visual acuity, dyschromatopsia, abnormal visual fields and optic nerve pallor, and negative screening tests including brain imaging and often extensive laboratory testing. Eighteen of the patients had a family history of optic neuropathy consistent with dominant transmission. All patients were negative for the $3460 \mathrm{~A}, 11778 \mathrm{~A}$, and $14484 \mathrm{C}$ primary LHON mutations. Informed consent was obtained from each study participant using an institutional IRB approved consent form.

\section{PCR and sequence analysis}

Blood samples were taken from the patients after obtaining informed consent. The genomic DNA was extracted from blood buffy coat using a QIAamp DNA blood kit (QIAGEN, Valencia, CA). All 28 exons of OPA1 gene, including exonintron junction, were amplified by PCR using coupled primers previously described in Toomes et al. ${ }^{5}$ The PCR reactions were carried out under standard conditions with $100 \mathrm{ng}$ genomic DNA and 10pmol coupled primers in $20 \mu \mathrm{l}$ of $1 \mathrm{x}$ PCR master mix (Promega, Madison, WI). The PCR products were gel purified with MinElute Gel Extraction Kit (QIAGEN Inc, Valencia, CA). Purified PCR products were sequenced using a CEQ8000 sequencer (CEQ DTCS-Quick Start Kit, Beckman Coulter, Fullerton, CA). Sequence visualization and assembly were performed using Sequencher and Chromas software and mutations were detected following alignment with normal OPA1 genomic or cDNA sequences (NCBI Blast).

\section{OPA1 mutation confirmation}

RFLP analysis was used to confirm the exon 27 (c.2780T $>$ A) and exon 28 (c.2883A $>$ C) mutations. Because these mutations did not alter an existing restriction site or create a new one, we used mismatched PCR primers to introduce a diagnostic, mutationspecific restriction site. ${ }^{22}$ For the c.2780T $>$ A mutation, a mismatched forward primer (5' - GCT GAA GAT GGT GAG AAG AAG ATT CAA T - $3^{\prime}$; mismatch bold) was used that generated an $M f e I$ site in the presence of the c.2780T>A mutation. Likewise, a mismatched PCR forward primer ( $5^{\prime}$ - TCA TTG AAG CTC TTC ATC AGG AGA AGT A - 3'; mismatch bold) was 
used to create a diagnostic TtaI site in the presence of the c. $2883 \mathrm{~A}>\mathrm{C}$ mutation. The digested products were run on $3 \%$ agarose gel.

Deletions in exon 17 (c.1654delT), exon 20 (c.1929delC) and exon 27 (c.2708 delTTAG) were confirmed by automated DNA sequencing of PCR-amplified individual cloned chromosomal fragments. The relevant portions of the OPA1 gene were cloned using pGEM-T Easy Vector System (Promega, Madison, WI), as described in Mayorov et al. ${ }^{24}$ Plasmid DNA concentration was determined by analytical gel and 10-20 ng DNA was used for sequencing. At least 24 individual clones were picked and grown for sequence analysis. A number of clones indicating the presence of deleted and normal OPA1 chromosomes were found in each case, thus verifying the mutation and heterozygosity.

\section{MtDNA haplotype analysis}

PCR-based RFLP assays were used to identify the nine (H, I, J, $\mathrm{K}, \mathrm{T}, \mathrm{U}, \mathrm{V}, \mathrm{W}$, and $\mathrm{X}$ ) mtDNA haplogroups that define the European haplogroups: $\mathrm{H}=-7052$ AluI, $-10394 D d e \mathrm{I}$; I $=-$ 1715DdeI, +8245AvaII, +10028AluI, +10394DdeI, J = $-13704 \mathrm{MvaI}$, +10394DdeI, -16065 Hinfl; $\mathrm{K}=-9052 \mathrm{HaeII}$, +12308 Hinfl, +10394 DdeI; $\mathrm{T}=+13366$ BamHI, +15606AluI; $\mathrm{U}=+12308$ Hinfl, $-10394 D d e \mathrm{I} ; \quad \mathrm{V}=-4577$ NlaIII; $-10394 D d e \mathrm{I} ; \mathrm{W}=+8249$ AvaII, -8994 HaeIII, -10394 DdeI; $\mathrm{X}=-1715$ DdeI, $-10394 D$ deI. ${ }^{25,26}$ All individuals not unambiguously testing positive for any of the above nine haplogroups were classified as "other."

\section{Statistical analysis}

To determine if the European mtDNA haplogroups are similarly distributed between controls and patient groups (either total ADOA patients or OPA1-negative ADOA patients), we used the Pearson $\chi^{2}$ statistics for an $m \times 2$ contingency table. Here, $m=$ number of haplogroups in the population. However, because the sample size is not large and thus some of the cells have a small number of individuals, the calculated $P$-value based on $\chi^{2}$ distribution is inaccurate. To accommodate this, we employed a Monte Carlo simulation procedure where we first simulate patients' haplogroups based on null hypothesis that patients have the same haplogroup distribution as controls. Then we calculate the Pearson $\chi^{2}$ statistics based on the simulated data and compare this value to the Pearson $\chi^{2}$ statistics based on the original data. This simulation is done 10,000 times, and the $P$-value is the proportion that the $\chi^{2}$ statistics from simulated data are greater than or equal to the $\chi^{2}$ statistics from original data.

The Monte Carlo simulation can also be used to test whether or not individual haplogroups are distributed similarly when comparing patient and control frequencies. However, since $2 \times 2$ tables are utilized in such analysis, we also used the Fisher's exact test. ${ }^{21}$ We calculated $P$-values based using both the simulation and Fisher's exact test methods. We use pSimulation and pFisher to indicate $P$-values calculated based on these two approaches, respectively, and in each case there was essentially no difference in $P$-values generated by the two methods.
Thus, in the Results section we report only the pFisher values for simplicity.

For statistical analysis, two groups of controls were used: the Southeastern United States regional controls $(n=246)^{26}$ and a global control group $(n=2645)$. Although the mtDNA haplogroup distribution in these two groups is essentially identical (no variation upon statistical comparison), the difference in sample size raised the possibility that the two groups would have yielded different statistical results when compared to our patient groups. Thus, we used both groups for data and statistical comparisons. Because the regional controls represent a more conservative (smaller sample size) statistical approach, we report $P$-values using this group of controls, except where clearly indicated in the text. For all statistical analysis, statistical significance was reached when $P<0.05$.

\section{RESULTS}

\section{OPA1 mutations in ADOA patients}

The complete sequence of all 28 OPA1 exons was obtained for 30 unrelated ADOA patients. Our methodology permitted the detection of all exon mutations as well as variants within the canonical splice site sequences. In total, we found 20 variants. Twelve of these are presumably polymorphisms since they have been previously reported in control chromosomes or are in intron sequences not related to a splice site junction (Table 2). Three of these (one silent substitution and two mis-

Table 1

OPA1 polymorphisms identified in ADOA patients

\begin{tabular}{|c|c|c|c|c|}
\hline DNA Change & Intron & $\begin{array}{c}\text { Number } \\
\text { of } \\
\text { Patients }\end{array}$ & Characteristics & References \\
\hline $\begin{array}{l}\text { Coding } \\
\text { Region }\end{array}$ & Exon & & & \\
\hline $\begin{array}{l}\text { c. } 2109 \mathrm{C}>\mathrm{T} \\
\text { (Ala; } \\
\text { silent) }\end{array}$ & 21 & 4 & Heterozygous & eOPAl \\
\hline $\begin{array}{l}\text { c.575C }>\text { T } \\
\quad \text { (Ala192Val) }\end{array}$ & 5 & 1 & Homozygous & eOPA1 \\
\hline $\begin{array}{l}\text { c. } 473 \mathrm{~A}>\mathrm{G} \\
\quad(\text { Asn158Ser })\end{array}$ & 4 & 6 & Heterozygous & eOPA1 \\
\hline Intron & Intron & & & \\
\hline c. $1+97 \mathrm{G}>\mathrm{A}$ & 1 & 1 & Heterozygous & New \\
\hline c. $6-13 \mathrm{~T}>\mathrm{G}$ & 6 & 1 & Heterozygous & New \\
\hline c. $8+4 \mathrm{~T}>\mathrm{C}$ & 8 & 13 & Heterozygous & eOPA1 \\
\hline c. $8+32 \mathrm{~T}>\mathrm{C}$ & 8 & 14 & Heterozygous & eOPA1 \\
\hline c. $11+92 \mathrm{~T}>\mathrm{A}$ & 11 & & & \\
\hline c. $15+24 \mathrm{~T}>\mathrm{A}$ & 15 & 3 & Heterozygous & New \\
\hline c. $15-33 \mathrm{C}>\mathrm{T}$ & 15 & 1 & Heterozygous & New \\
\hline c. $26+12 \mathrm{~T}>\mathrm{G}$ & 26 & 1 & Heterozygous & New \\
\hline c. $26+25 \mathrm{~T}>\mathrm{A}$ & 26 & 13 & Homozygous & eOPAl \\
\hline c. $27-5 \mathrm{C}>\mathrm{T}$ & 27 & 2 & Heterozygous & New \\
\hline
\end{tabular}


Table 2

Novel, heterozygous OPAl mutations found in ADOA patients

\begin{tabular}{|c|c|c|c|c|c|}
\hline DNA Change $^{a}$ & $\begin{array}{c}\text { Patient } \\
\text { Number }\end{array}$ & Exon & $\begin{array}{l}\text { Number of } \\
\text { Families }\end{array}$ & $\begin{array}{l}\text { Amino Acid } \\
\text { Change }\end{array}$ & Protein Domain ${ }^{b}$ \\
\hline \multicolumn{6}{|l|}{ Mis-sense mutation } \\
\hline c. $22 \mathrm{G}>\mathrm{T}$ & 1 & 1 & 1 & Ala8Ser & Mitochondrial targeting sequence \\
\hline c. $239 \mathrm{~A}>\mathrm{G}$ & 2 & 2 & 1 & Tyr80Cys & Mitochondrial targeting sequence \\
\hline c. $2522 \mathrm{~A}>\mathrm{G}$ & 3 & 25 & 1 & Tyr841Cys & C-terminal \\
\hline \multicolumn{6}{|l|}{ Non-sense mutation } \\
\hline c. $2780 \mathrm{~T}>\mathrm{A}$ & 4 & 27 & 1 & Leu927Stop & Coiled-coil \\
\hline c. $2883 \mathrm{~A}>\mathrm{C}$ & 5 & 28 & 2 & Stop961Tyr & Coiled-coil \\
\hline \multicolumn{6}{|l|}{ Deletion } \\
\hline c.1654delT & 6 & 17 & 1 & Trp552fs & Dynamin central region \\
\hline c.1929delC & 7 & 20 & 1 & Thr643fs & Dynamin central region \\
\hline c.2708del(TTAG) & 8 & 27 & 1 & Val903fs & Coiled-coil \\
\hline
\end{tabular}

${ }^{a}$ Nucleotide designation beginning 1 at position 56 (translation start) of GenBank entry AB011139.

${ }^{b}$ From references 3 and 4.

fs, frameshift.

sense mutations) single nucleotide polymorphisms (SNPs) were found in exon sequences and have been previously reported in the literature and in the database for OPA1 mutations, eOPA1.27 Of the nine intron SNPs, six have not been previously described and therefore represent new SNPs.

Eight mutations were found that alter the OPA1 protein (Table 3) and seven of these are novel. All of these mutations were heterozygous, were not found in 100 control chromosomes, and are absent from the genetic database for OPA1 pathogenic and polymorphic variants, eOPA $1 .{ }^{27}$ The c. $22 \mathrm{G}>\mathrm{T}$ (ala8ser; exon 1), c.239A $>\mathrm{G}$ (tyr80cys; exon 2), and c.2522G >A (tyr841cys; exon 25) are mis-sense mutations that are either in the mitochondrial targeting signal and/or alter phylogenetically conserved amino acids (Table 3). The c.2780T $>$ A (leu927stop) and the c.2883 (stop961tyr) mutations create or eliminate stop codons in exons 27 and 28 respectively. These protein length-altering mutations were confirmed by RFLP analysis since the $c .2780 \mathrm{~T}>\mathrm{A}$ mutation eliminates an $\mathrm{MfeI}$ site and the c.2883A $>\mathrm{C}$ variant creates a TtaI site (data not shown). While the c.2780T $>$ A mutation eliminates 33 amino acids from the C-terminal end of the OPA1 gene product, the c.2883A $>\mathrm{C}$ mutation extends the OPA1 polypeptide by three (Tyr-Ile-Leu-stop) amino acids. Hence, this mutation represents the first ADOA-associated mutation to extend the OPA1 protein. Three microdeletions were found in exons 17, 20, and 27, all of which caused a translational frameshift resulting in a premature stop codon (Table 4). One of these deletions, a four-base-pair deletion at nucleotide 2708 in exon 27 has been previously reported in the literature and in fact may be a hotspot for recurrent ADOA-associated OPA1 mutations. ${ }^{15}$ The three frameshift mutations were confirmed by cloning and sequence analysis of individual clones (data not shown).
The clinical characteristics of our eight ADOA patients harboring unique OPA1 mutations are indicated in Table 5. All patients had a childhood age of onset and five patients (63\%) had a positive family history. For all patients, visual acuity was reduced and bilateral optic disc pallor was present. In all but two patients, visual field testing indicated bilateral central or cecocentral scotomas.

Table 3

OPA1 Mis-Sense Mutations Alter Conserved Amino Acids

\begin{tabular}{lcccccccccc}
\hline c.239G mutation; tyrosine to cysteine at exon 2 amino acid & $80^{*}$ & & \\
\hline Patient & S & P & I & K & C & G & Y & Q & P \\
H. sapiens & S & P & I & K & Y & G & Y & Q & P \\
M. muscularis & S & P & I & K & Y & G & Y & Q & P \\
G. gallus & L & N & V & K & Y & G & Y & Q & S \\
D. melanogaster & P & P & P & S & R & G & Y & - & - \\
A. gambiae & - & - & - & - & Y & G & G & Q & Q
\end{tabular}

c.2522G mutation; tyrosine to cysteine at exon 25 amino acid 841

\begin{tabular}{lccccccccc}
\hline Patient & W & H & Q & V & C & R & R & H & F \\
H. sapiens & W & H & Q & V & Y & R & R & H & F \\
M. muscularis & W & H & Q & V & Y & R & R & H & F \\
G. gallus & W & H & Q & I & Y & R & R & Y & F \\
D. melanogaster & W & F & P & V & Y & R & K & H & F \\
A. gambiae & W & Y & P & I & Y & R & R & H & F \\
C. elegans & W & K & L & V & F & K & K & H & F \\
\hline
\end{tabular}

M. muscularis, mouse; G. gallus, chicken; D. melanogaster, fruit fly; A. gambiae, mosquito. 
Table 4

Clinical features of patients with new ADOA-associated OPA1 mutations

\begin{tabular}{|c|c|c|c|c|c|c|c|c|}
\hline Patient & Mutation & $\begin{array}{l}\text { Patient } \\
\text { Age }^{a}\end{array}$ & $\begin{array}{l}\text { Age of } \\
\text { Onset }^{b}\end{array}$ & Gender/Race & $\begin{array}{l}\text { Family History of } \\
\text { Optic Neuropathy }\end{array}$ & $\begin{array}{l}\text { Visual } \\
\text { Acuity }^{a}\end{array}$ & Visual Field $^{a}$ & Fundus $^{a}$ \\
\hline 1 & c. $22 \mathrm{G}>\mathrm{T}$ & 49 & Childhood & $\mathrm{M} / \mathrm{W}$ & $\mathrm{Y}$ & $\begin{array}{l}20 / 70 \text { OD } \\
20 / 60 \text { OS }\end{array}$ & $\begin{array}{l}\text { Cecocentral } \\
\text { scotoma OU }\end{array}$ & Optic nerve pallor OU \\
\hline 2 & c. $239 \mathrm{~A}>\mathrm{G}$ & 38 & Childhood & $\mathrm{F} / \mathrm{AA}$ & $\mathrm{N}$ & $\begin{array}{l}20 / 70 \text { OD } \\
20 / 70 \text { OS }\end{array}$ & $\begin{array}{l}\text { Central } \\
\text { scotoma OU }\end{array}$ & Temporal pallor OU \\
\hline 3 & c. $2522 A>G$ & 6 & 4 years & $\mathrm{F} / \mathrm{AA}$ & $\mathrm{Y}$ & $20 / 100 \mathrm{OU}$ & $\begin{array}{l}\text { Central } \\
\text { scotoma OU }\end{array}$ & Optic nerve pallor OU \\
\hline 4 & c. $2780 \mathrm{~T}>\mathrm{A}$ & 12 & 6 years & $\mathrm{M} / \mathrm{W}$ & $\mathrm{Y}$ & $\begin{array}{l}20 / 40 \text { OD } \\
20 / 40 \text { OS }\end{array}$ & Full & Temporal pallor OU \\
\hline 5 & c. $2883 A>C$ & 26 & $5-6$ years & F/AA & $\mathrm{N}$ & $\begin{array}{l}20 / 100 \text { OD } \\
20 / 200 \text { OS }\end{array}$ & $\begin{array}{l}\text { Cecocentral } \\
\text { scotoma OU }\end{array}$ & $\begin{array}{l}\text { Temporal pallor OU } \\
\quad \text { (excavated) }\end{array}$ \\
\hline 6 & c.1654delT & 37 & 16 years & $\mathrm{M} / \mathrm{W}$ & $\mathrm{N}$ & $20 / 40 \mathrm{OU}$ & $\begin{array}{l}\text { Centrocecal } \\
\text { depression OS }\end{array}$ & $\begin{array}{l}\text { Temporal pallor OU } \\
\quad \text { (excavated) }\end{array}$ \\
\hline 7 & c.1929delC & 60 & Childhood & $\mathrm{F} / \mathrm{W}$ & $\mathrm{Y}$ & $\begin{array}{l}20 / 200 \text { OD } \\
20 / 200 \text { OS }\end{array}$ & $\begin{array}{l}\text { Cecocentral } \\
\text { scotoma OU }\end{array}$ & $\begin{array}{l}\text { Temporal pallor OU } \\
\quad \text { (excavated) }\end{array}$ \\
\hline 8 & c.2708del(TTAG) & 7 & 6 years & F/W & $\mathrm{Y}$ & $\begin{array}{l}20 / 100 \text { OD } \\
20 / 100 \text { OS }\end{array}$ & $\begin{array}{l}\text { Cecocentral } \\
\text { scotoma OU }\end{array}$ & Temporal pallor OU \\
\hline
\end{tabular}

${ }^{a}$ Data collected at primary presentation to Neuro-Ophthalmology Unit, Emory University School of Medicine.

${ }^{b}$ Age of onset of visual loss corresponds to when a subnormal visual acuity was noted (either during a screening vision test or when the patient noticed visual changes). M, male; F, female; C, Caucasian; AA, African American; Y, yes; N, no.

Seven of the novel OPA1 mutations were present in a single patient or family. The c.2883A $>$ C mutation, however, was found in two (patients 2 and 5; Table 4) patients, one of which harbored only this variant and the other (Patient 2; Table 4) also containing the c.239A $>\mathrm{G}$ missense mutation in exon 2, indicating compound heterozygosity for Patient 2. Interestingly, this patient exhibited a typical clinical presentation among our OPA1-positive patients, although there is no family history of optic atrophy. Overall, despite the discovery of 8 pathogenic OPA1 variants, we found such variants in $7 / 30$
(23\%) of our ADOA patients using our approach of genomic sequencing of exons and splice junctions.

\section{MtDNA haplotypes in ADOA patients}

We performed mtDNA haplotype analysis on 29 Caucasian ADOA patients. We were able to assign all but one patient to one of the nine mtDNA lineages (haplogroups $\mathrm{H}, \mathrm{I}, \mathrm{J}, \mathrm{K}, \mathrm{T}, \mathrm{U}, \mathrm{V}, \mathrm{W}$ and $\mathrm{X}$ ) that account for essentially all European-derived individuals (Table 5). We then compared the distribution of mtDNA lineages between controls (using both 2645 global Caucasian con-

Table 5

MtDNA haplogroups in caucasian ADOA patients

\begin{tabular}{|c|c|c|c|c|c|}
\hline $\begin{array}{l}\text { MtDNA } \\
\text { Haplogroup }\end{array}$ & $\begin{array}{l}\text { Frequency in Caucasian } \\
\text { Controls }^{a}(\%)\end{array}$ & $\begin{array}{c}\text { Frequency in Regional } \\
\text { Caucasion } \\
\text { Controls }^{b}(\%)\end{array}$ & $\begin{array}{l}\text { Total ADOA Pts } \\
\quad(n=29)\end{array}$ & $\begin{array}{l}\text { OPA1-Positive } \\
\text { ADOA Pts } \\
(n=5)\end{array}$ & $\begin{array}{c}\text { OPA1-Negative } \\
\text { ADOA Pts } \\
(n=24)\end{array}$ \\
\hline $\mathrm{H}$ & 43 & 44 & 11 & 38 & 4 \\
\hline I & 3 & 4 & 3 & 10 & - \\
\hline J & 9 & 8 & 7 & 24 & - \\
\hline K & 9 & 9 & - & - & - \\
\hline $\mathrm{T}$ & 10 & 12 & 2 & 7 & - \\
\hline $\mathrm{U}$ & 13 & 10 & 4 & 14 & 1 \\
\hline V & 3 & 2 & - & - & - \\
\hline W & 2 & 2 & - & - & - \\
\hline $\mathrm{X}$ & 2 & 1 & 1 & 3 & - \\
\hline Other & 6 & 8 & 1 & 3 & - \\
\hline
\end{tabular}

${ }^{a} 2,645$ Caucasian controls. $22,23,29,31,40-42$

${ }^{b} 246$ Southeastern United States Caucasians from reference 26. 
trols from the literature and also 246 regional Caucasian controls from the Southeastern United States, as described in Booker et al. ${ }^{26}$ and ADOA patients (total patient group and OPA1-negative patients). OPA1-positive patients were not included in the comparative analysis since only five of our OPA1-positive patients were Caucasian, thus severely limiting statistical power. P values, except where noted, are given for comparisons using the more statistically conservative (due to smaller sample size and reduced population substructure) regional control group.

We first compared the overall distribution of mtDNA haplotypes and found that the two distributions differ when regional controls are compared to either the total patient $(P=0.0282)$ or the OPA1-negative $(P=0.0085)$ patient groups. We next asked if specific haplogroups were over- or under-represented in the ADOA patients. Haplogroups I and $J$ were found to be more common in ADOA patients than expected by chance (Table 5). Haplogroup J, for example, is roughly three-fold overrepresented when our total (pFisher's $=0.0136$ ) or OPA1-negative (pFisher's = 0.0072) Caucasian ADOA patients were compared with regional controls. This difference was also statistically significant upon comparison to the global controls ( $\mathrm{pFishers}=0.0193$ for comparison with total patient group; pFishers $=0.0066$ for comparison with OPA1-negative patient group). Haplogroup I is also found 3-4 times more frequently in Caucasian total or OPA1-negative ADOA patients than expected, although this difference only reached statistical significance when compared to the global control group (pFisher's $=0.0345$ and 0.0210 , respectively).

Haplogroup K was under-represented in our patients, as it was not found in any ADOA patient despite being present in $9 \%$ of all Caucasian mtDNAs in the United States and worldwide. This distribution difference, however, did not meet statistical significance. No other mtDNA haplogroup deviated from the expected when comparing patients with controls.

When considering the five Caucasian patients positive for a pathogenic OPA1 mutation, four (80\%) patients were found to have a haplogroup $\mathrm{H}$ mtDNA background, the most common mtDNA lineage in European-derived individuals, being found in $43 \%$ of European and North American Caucasian control populations. ${ }^{23,25}$ The remaining OPA1 mutation-positive patient belonged to mtDNA haplogroup I. There was no obvious association between mtDNA haplotype and family history when considering total ADOA patients, OPA1 mutation-positive patients, or OPA1 mutation-negative patients.

\section{DISCUSSION}

\section{Novel OPA1 mutations in ADOA}

OPA1 exon sequence analysis from $30 \mathrm{ADOA}$ patients revealed seven new coding region variants that likely cause optic atrophy. Six of these have not been previously reported in the literature, thus bringing the total number of ADOA-associated OPA1 mutations to $105 .{ }^{3,27}$ To date, $\mathrm{ADOA}$-associated mutations tend to cluster within three OPA1 functional domains: the $\mathrm{N}$-terminal mitochondrial leader sequence (exons 1 and 2), the conserved
GTP binding domain (exons 8-16), and the C-terminal coiledcoil motif (exons 27 and 28). ${ }^{15,27}$ The distribution of the novel OPA1 mutations reported here is consistent with this observation in that two of our variants are within the leader sequence (thus doubling the total number of reported pathogenic leader sequence mutations found in patients) and three fall within the Cterminal coiled-coil region. ${ }^{3,27}$

Nearly one-quarter of our patients harbored a deleterious OPA1 mutation. This is consistent with, although slightly lower than, the detection frequencies of $28-49 \%$ found in the literature. ${ }^{5,15,16,28}$ Reasons for this could be technical, as we only sequenced exon sequences from DNA, meaning that certain RNA expression or processing mutations may have escaped our detection. However, all known ADOA-associated OPA1 mutations have been found either within exons ( $82 \%$ of all known mutations) or within 10 nucleotides of the $5^{\prime}$ or $3^{\prime}$ splice junction. ${ }^{27}$ Our method, therefore, would have detected all known pathogenic OPA1 mutations. Further, two of our patients harbored intron mutations (that were not known polymorphisms) within 15 nucleotides of an intron-exon border (see Table 1). For these patients, we performed cDNA analysis to detect RNA splicing defects and none were found (data not shown). Thus, although it remains possible that a fraction of our OPA1-negative patients contain a RNA processing or expression mutation, we are reasonably confident that our OPA1-negative patients do not harbor disease-causing OPA1 mutations. It is also possible that genetic heterogeneity accounts for the modest mutation detection rate, as four other genetic loci (OPA2, OPA3, OPA4, and OPA5) have been mapped that have been associated with Mendelian transmission of optic atrophy. ${ }^{17-19}$ Also, the majority of the OPA1 mutations reported here were found in probands with a family history of ADOA, thus supporting the contention that OPA1 mutations are most often found in families with more than one affected individual. ${ }^{16}$ However, singleton cases are not uncommon in ADOA.5,15,16 Such cases may represent true incomplete penetrance or variable clinical expression in that other family members that harbor a pathogenic OPA1 mutation show subtle clinical signs only detectable by careful clinical examination. ${ }^{3}$ Alternatively, sporadic cases may be more likely to result from mutation in other genes that can cause bilateral optic atrophy.

Consistent with the literature, two-thirds of the new OPA1 mutations reported here result in either a truncated gene product or alter the translational stop codon. Of particular interest is the c.2883C $>\mathrm{T}$ exon 28 transition. This mutation changes the normal OPA1 stop codon at the end of exon 28 to a tyrosine codon resulting in an extension of the OPA1 polypeptide by three amino acids (Tyr-Ile-Leu-STOP). According to Thisetlton et al., this alteration could disrupt the coiled-coil domain predicted by exon 28 amino acids $940-961 .{ }^{15}$ Coiled-coil motifs are believed to be involved in protein-protein interactions ${ }^{29}$ and this domain is essential for proper OPA1 activity and mitochondrial fusion. ${ }^{6}$ This variant likely causes ADOA since it alters a polypeptide region predicted to be important for protein-protein interactions, has not been found in control chromosomes, and localizes to a region that is not only a "hotspot" 
for ADOA mutations but has been shown to be essential for OPA1 function. Interestingly, this mutation is the first variant reported to extend the length of the OPA1 polypeptide by alteration of the stop codon and is the only OPA1 variant that we found in two independent patients.

The preponderance of translation termination mutations tends to support a genetic mechanism featuring haploinsufficiency where the absence of one allele is sufficient for disease expression. However, as pointed out by Thistelton et al., a number of mis-sense mutations are unambiguously associated with the disease, leaving the question of pathogenetic mechanism(s) open. ${ }^{15}$ In this report, for example, we found three novel mis-sense mutations. The first, c.22G $>$ T, clearly occurs within the mitochondrial targeting leader motif as it alters the alanine found at exon 1 amino acid eight to a serine. Mitochondrial targeting sequences are characterized by amphiphilic $\alpha$-helices with an overall positive net charge. The non-polar alanine is a common and preferred amino acid in such signals, while serine is considered anti- $\alpha$-helix. Hence, this mutation may disrupt the targeting of the OPA1 gene product to the mitochondria. Similarly, the c.239A $>$ G mutation replaces a tyrosine with a cysteine at amino acid 80 of exon 2 . This variant alters a putative cleavage site (amino acid residues 71-80) within the mitochondrial leader sequence and therefore may prevent the proper importation of the OPA1 protein. Although, in the absence of functional data, the pathogenicity of mis-sense mutations is difficult to prove, we feel that these two mutations are etiologically relevant to ADOA since they are heterozygous variants not found in our controls nor in the literature and the amino acid changes alter key components of the mitochondrial leader signal peptide.

One of our patients (Patient 2; Table 4) harbored both the c. $239 \mathrm{~A}>\mathrm{G}$ missense mutation in exon 2 and the c.2883A $>\mathrm{C}$ mutation in exon 28 and is thus a compound heterozygote. Pesch et al. described the only other compound heterozygous patient. ${ }^{16}$ In that case, the patient had a more severe clinical phenotype than did heterozygous affected siblings and parents. Our case is different in that our patient has no apparent family history of optic atrophy or other eye disease. Further, our patient exhibits typical clinical signs for ADOA, including a childhood age of onset. The compound heterozygous patient described by Pesch et al. harbored two mis-sense mutations in exon 8 , suggesting that, individually, the exon 8 variants are relatively mild, but when combined precipitate a more severe clinical course. ${ }^{16}$ Our patient contained an exon 2 mis-sense mutation in the mitochondrial targeting sequence and an exon 28 mutation that disrupts a coiled-coil domain and extends the OPA1 protein by three amino acids. In our case, it may also be true that these two mutations are relatively mild, but are not deleteriously synergistic when found in combination. Overall, then, our data suggests that haploinsufficiency may well be the dominant pathogenetic mechanism observed in OPA1-associated ADOA, although alternate mechanisms (dominant negative, semi-dominant) remain possible.

\section{MtDNA haplotypes as a genetic modifier}

To test the hypothesis that mtDNA background serves as a genetic modifier for OPA1-positive and/or OPA1-negative ADOA clinical expression, we determined the mtDNA haplotype for Caucasian ADOA patients. Nine different mtDNA lineages-H, I, J, U, K, T, W, X, and V -comprise nearly all European mtDNAs. Although $80 \%$ of our OPA1-positive patients harbored haplogroup $\mathrm{H}$, a detailed and statistically reliable analysis of mtDNA background in this patient group was prohibited by the low number of patients. For our total and OPA1negative patient groups, our results show that overall, the nine Caucasian mtDNA haplogroups were distributed differently in ADOA patients in comparison to controls. Further, we observed that individual mtDNA lineage J was consistently and significantly over-represented in our two ADOA patient groups relative to controls.

The finding of an mtDNA lineage in higher-than-expected frequencies in patient populations may indicate an association that is etiologically relevant. In this case, polymorphisms defining haplogroup J may interact with the primary (and as yet undefined) genetic lesion in OPA1-negative ADOA in a way that increases the probability of optic atrophy. Such a mechanism has long been postulated for LHON, where haplogroup J apparently influences the expression of certain LHON mtDNA mutations. For example, haplogroup J is associated with nearly $75 \%$ of 14484 C LHON cases $^{21,23,30}$ and is exclusively associated with the 10663C LHON mutation..$^{22}$ Presumably, haplogroup J polymorphisms facilitate the deleterious effects of these primary LHON mutations, although the polymorphisms are subclinical in the absence of the $14484 \mathrm{C}$ or $10633 \mathrm{C}$ mutations. Haplogroup J has also been implicated as a genetic modifier for a number of other disorders including Parkinson disease, ${ }^{31-34}$ multiple sclerosis with and without optic neuritis, ${ }^{35,36}$ and aging. ${ }^{37-39}$ For LHON, the mutation-haplogroup associations appear real, but the origins of any association as well as the molecular mechanisms describing such genomic interactions remain unclear.

If a similar mechanism held true for ADOA, the genotypic interaction could be synergistic but indirect in that the haplogroup J mtDNA background may modestly alter mitochondrial metabolism (e.g., bioenergetics, oxidative stress or apoptosis) in such a way to facilitate ADOA expression in OPA1-negative patients. In other words, haplogroup J polymorphisms may provide a biochemical environment that facilitates non-OPA1-mediated ADOA. Alternatively, a direct interaction between the ADOA gene product and the haplogroup J-prescribed mtDNA polypeptide(s) is also possible, particularly if the mutant gene product in OPA1-negative ADOA is targeted to the mitochondria (like OPA1). One potential outcome of this scenario is that ADOA penetrance may be higher in OPA1 mutation-negative families harboring a high proportion of haplogroup J mtDNAs. Such an association would not necessarily be readily apparent without DNA testing, as females harboring other mtDNA haplotypes marry into families. Unfortunately, our families are not large enough to draw valid conclusions regarding this issue. Overall, 
however, it remains possible that ADOA, being a mitochondrial disease similar in neuropathology to LHON, may employ a complex genetic mechanism in a subset of cases that features mtDNAnDNA interactions that alter mitochondrial biology. This, in turn, might explain some of the observed variation in penetrance and expressivity in ADOA, both within and between ADOA families. Importantly, it should be noted that mtDNA background cannot account for clinical variation in some ADOA families, as siblings harboring the identical mtDNA and ADOA nuclear mutation can have different clinical pictures. Thus, if mtDNA genetic background is relevant in ADOA, it accounts for only a portion of the observed variation.

Although our results did not meet statistical significance, haplogroup $\mathrm{K}$ was absent in all of our ADOA patients despite accounting for $9 \%$ of all Caucasian mtDNAs. This raises the possibility that this mtDNA lineage is protective regarding OPA1-negative ADOA. Such a scenario involving haplogroup $\mathrm{K}$ has been previously postulated for another neurological disease featuring mitochondrial dysfunction, namely Parkinson disease (PD), where it has been associated with a reduced risk for PD in an Italian study ${ }^{40}$ and an American study. ${ }^{32}$ The latter study concluded that the $10398 \mathrm{G}$ polymorphism found in haplogroup $\mathrm{K}$ mtDNAs was strongly associated with this protective effect. Interestingly, in European-derived individuals, the $10398 \mathrm{G}$ polymorphism is found only in haplogroups I, J, and $\mathrm{K}$ : the three lineages that exhibit unexpected frequencies in ADOA patients. However, because haplogroups I and J are over-represented - and haplogroup $\mathrm{K}$ is under-represented in our ADOA patients, it is unlikely that the $10398 \mathrm{G}$ polymorphism has a role in our observations, consistent with the findings of Ghezzi et al. in PD. ${ }^{40}$ As pointed out by Autere et al., the KU haplogroup cluster contains the lowest average amount of amino acid substitutions among complex I genes. ${ }^{33}$ Because accumulated polymorphisms that result in amino acid substitutions may alter mitochondrial bioenergetic flux or production of oxygen radicals, the lack of polymorphic mis-sense mutations may be beneficial in many molecular settings, potentially even reducing a risk for neuronal damage in certain mitochondrial disorders. In this context, haplogroup K could be protective.

Our results suggest that certain mtDNA backgrounds may have a role in modulating the expression of OPA1-negative ADOA. However, caution is warranted regarding this conclusion due to the moderate number of ADOA patients in the study and the possibility of false association due to population substructure or other confounders. We have tried to minimize the effects of population substructure on our comparative and statistical analysis by utilizing a region-matched control group, thus homogenizing our patient and control groups by reducing or eliminating the possibility of regional-, population-, or continent-specific variation in Caucasian mtDNA haplogroup frequencies. Also, because ADOA is typically an early-onset disease without gender bias, cases are generally apparent within the general population. Hence, we should have a reasonably well-delineated patient cohort (with little ambiguity regarding affected status) along with an appropriate control group. Nevertheless, these results must be considered preliminary until substantiated by other independent investigations. Finally, our observed associations pertain only to OPA1-negative ADOA patients. Thus, any detailed genotype/phenotype analysis must await the elucidation of the gene(s) responsible for OPA1-negative ADOA.

\section{ACKNOWLEDGMENTS}

This study was supported by grants R01 EY014393 (MDB) and P30 EY06360 (NJN) from the National Institutes of Health and by a departmental grant (Department of Ophthalmology, EUSM) from Research to Prevent Blindness, Inc., New York, New York. Dr Newman is a recipient of a Research to Prevent Blindness Lew R. Wasserman Merit Award.

\section{References}

1. Johnston PB, Gaster RN, Smith VC, Tripathi RC. A clinico-pathological study of autosomal dominant optic atrophy. Am J Ophthalmol 1979;88:868-875.

2. Carelli V, Ross-Cisneros FN, Sadun AA. Mitochondrial dysfunction as a cause of optic neuropathies. Prog Ret Eye Res 2004;23:53-89.

3. Puomila A, Huoponen K, Mantyjarvi M, Hamalainen P, et al. Dominant optic atrophy: correlation between clinical and molecular genetic studies. Acta Ophthalmol Scand 2005;83:337-346.

4. Delettre C, Lenaers G, Griffoin J-M, Gigarel N, et al. Nuclear gene, OPA1, encoding a mitochondrial dynamin-related protein, is mutated in dominant optic atrophy. Nat Genet 2001;26:207-210.

5. Toomes C, Marchbank NJ, Mackey DA, Craig JE, et al. Spectrum, frequency and penetrance of OPAl mutations in dominant optic atrophy. Hum Mol Genet 2001; 10:1369-1378.

6. Cipolat S, Martins de Brit O, Dal Zili B, Scorrano L. OPA1 requires mitofusin 1 to promote mitochondrial fusion. Proc Natl Acad Sci U S A 2004;101:15927-15932.

7. Delettre C, Griffoin J-M, Kaplan J, Dollfus H, et al. Mutation spectrum and splicing variants in the OPAl gene. Hum Genet 2001;109:584-591.

8. Alexander C, Votruba M, Pesch UEA, Thiselton DL, et al. OPAl, encoding a dynamin-related GTPase, is mutated in autosomal dominant optic atrophy linked to chromosome 3q28. Nat Genet 2000;26:211-215.

9. Aijaz S, Erskine L, Jeffery G, Bhattacharya SS, et al. Developmental expression profile of the optic atrophy gene product: OPAl is not localized exclusively in the mammalian retinal ganglion cell layer. IVOS 2004;45:1667-1673.

10. Olichon A, Emorine LJ, Descoins E, Pelloquin L, et al. The human dynamin-related protein OPAl is anchored to the mitochondrial inner membrane facing the intermembrane space. FEBS 2002Let;523:171-176.

11. Griparic L, van der Wel NN, Orozco IJ, Peters PJ, et al. Loss of intermembrane space protein Mgm1/OPAl induces swelling and localized constrictions along the lengths of mitochondria. J Biol Chem 2004;279:18792-18798.

12. Olichon A, Baricault L, Gas N, Guillou E, et al. Loss of OPAl perturbates the mitochondrial inner membrane structure and intergrity, leading to cytochrome c release and apoptosis. J Biol Chem 2003;278:7743-7746.

13. Lee Y-J, Jeong S-Y, Karbowski M, Smith CL, et al. Roles of the mammalian mitochondrial fission and fusion mediators Fis1, Drp1, and OPA1 in apoptosis. Mol Bio Cell 2004;5:5001-5011.

14. Lodi R, Tonon C, Valentino ML, Iotti S, et al. Deficit of in vivo mitochondrial ATP production in OPA1-related dominant optic atrophy. Ann Neurol 2004;56:719-723.

15. Thiselton DL, Alexander C, Taanman J-W, Brooks S, et al. A comprehensive survey of mutations in the OPAl gene in patients with autosomal dominant optic atrophy. Invest Ophthalmol Vis Sci 2002;43:1715-1724.

16. Pesch UE, Leo-Kottler B, Mayer S, Jurklies B, et al. OPAl mutations in patients with autosomal dominant optic atrophy and evidence for semi-dominant inheritance. Hum Mol Genet 2001;10:1359-1368.

17. Votruba M. Molecular genetic basis of primary inherited optic neuropathies. Eye 2004;18:1126-1132.

18. Reynier P, Amanti-Bonneau P, Verny C, Olichon A, et al. OPA3 gene mutations responsible for autosomal dominant optic atrophy and cataract. J Med Gen 2004;41: el10. 
19. Barbet F, Hakiki S, Orssaud C, Gerber S, et al. A third locus for dominant optic atrophy on chromosome 22q. J Med Gen 2005;42:e1.

20. Newman NJ, Biousse V. Hereditary optic neuropathies. Eye 2004;18:1144-1160.

21. Brown MD, Sun F, Wallace DC. Clustering of Caucasian Leber Hereditary Optic Neuropathy patients containing the 11778 or 14484 mutations on a mtDNA lineage. Am J Hum Genet 1997;60:381-387.

22. Brown MD, Starikovskaya E, Derbeneva O, Hosseini S, et al. The role of mtDNA background in disease expression: a new primary LHON mutation associated with Western Eurasian haplogroup. J Hum Genet 2002;110:130-138.

23. Torroni A, Petrozzi M, D’Urbano L, Selitto D, et al. Haplotype and phylogenetic analyses suggest that one European-specific mtDNA background plays a role in the expression of LHON by increasing the penetrance of the primary mutations 11778 and 14484. Am J Hum Genet 1997;60:1107-1121.

24. Mayorov VI, Biousse V, Newman NJ, Brown MD. The role of the ND5 gene in LHON: Characterization of a new, heteroplasmic LHON mutation. Ann Neurol 2005;58:807-811.

25. Finnila S, Hassenin SN, Ala-Kokka M, Majamaa K. Phylogenetic network of the mtDNA haplogroup U in Northern Finland based on sequence analysis of the complete coding region by conformation-sensitive gel electrophoresis. Am J Hum Genet 2000;66:1017-1026.

26. Booker LM, Habermacher GM, Jessie BC, Sun QC, et al. North American Caucasian mitochondrial haplogroups in prostate and renal cancer. J Urol 2006;172:468-472.

27. Ferre M, Amati-Bonneau P, Tourmen Y, Malthiery Y, et al. eOPA1: an online database for OPAl mutations. Hum Mutat 2005;25:423-428.

28. Baris O, Delettre C, Amati-Bonneau P, Surget M-O, et al. Fourteen novel OPA1 mutations in autosomal dominant optic atrophy including two de novo mutations in sporadic optic atrophy. Hum Mutat 2003;21: 656-662.

29. Lupas A. Predicition and analysis of coil-coiled structures. Methods Enzymol 1996; 266:513-525

30. Brown MD, Torroni A, Reckord CL, Wallace DC. Phylogenetic analysis of Leber's Hereditary Optic Neuropathy mitochondrial DNAs indicates multiple independent occurrences of the common mutations. Hum Mutat 1995;6:311-325.

31. Ross OA, McCormack R, Maxwell LD, Duguid RA, et al. mt4216C variant in linkage with the mtDNA TJ cluster may confer a susceptibility to mitochondrial dysfunction resulting in an increased risk of Parkinson's disease in the Irish. Exp Gerontol 2003;38:397-405.
32. Van der Walt JM, Nicodemus KK, Martin ER, Scott WK, et al. Mitochondrial polymorphisms significantly reduce the risk of Parkinson disease. Am J Hum Genet 2003;72:804-811.

33. Autere J, Moilanen JS, Finnila S, Soininen H, et al. Mitochondrial DNA polymorphisms as risk factors for Parkinson's disease and Parkinson's disease dementia. Hum Genet 2004;15:29-35.

34. Huerta C, Castro MG, Coto E, Blazquez M, et al. Mitochondrial DNA polymorphisms and risk of Parkinson's disease in Spanish population. J Neurol Sci 2005;236: 49-54.

35. Kalman B, Li S, Chatterjee D, O'Connor J, et al. Large scale screening of the mitochondrial DNA reveals no pathogenic mutations but a haplotype associated with multiple sclerosis in Caucasians. Acta Neurol Scand 1999;99:16-25.

36. Reynier P, Penisson-Besnier I, Moreau C, Savagner F, et al. mtDNA haplogroup J: a contributing factor of optic neuritis. Eur J Hum Genet 1999;3:404-406.

37. De Benedictis G, Rose G, Carrierri G, De Luca M, et al. Mitochondrial DNA inherited variants are associated with successful aging and longevity in humans. FASEB J 1999;13:1532-1536.

38. Niemi A-K, Hervonen A, Hurme M, Karhunen PJ, et al. Mitochondrial DNA polymorphisms associated with longevity in a Finnish population. Hum Genet 2003;112: 29-33.

39. Dato S, Passarino G, Ros G, Altomare K, et al. Association of the mitochondrial DNA haplogroup J with longevity is population specific. Eur J Hum Genet 2004;12: 1080-1082.

40. Ghezzi D, Marelli C, Achilli A, Goldwurm S, et al. Mitochondrial DNA haplogroup $\mathrm{K}$ is associated with a lower risk of Parkinson's disease in Italians. Eur J Hum Genet 2005;13:748-752.

41. Herrnstadt C, Elson JL, Fahey E, Preston G, et al. Reduced-median-network analysis of complete mitochondrial DNA coding-region sequences for the major African, Asian, and European haplogroups. Am J Hum Genet 2002;70:11521171.

42. Allard MW, Miller K, Wilson M, Monson K, et al. Characterization of the Caucasian haplogroups present in the SWGDAM forensic mtDNA dataset for 1771 human control region sequences. J Forensic Sci 2002;47:1215-1223. 